\title{
Validez predictiva de las competencias socioemocionales sobre la resiliencia en adolescentes mexicanos*
}

\author{
Predictive validity of socio-emotional skills \\ on the resilience of Mexican adolescents
}

Norma Alicia Ruvalcaba Romero

ORCID: 0000-0001-9209-8751

Universidad de Guadalajara, México

Julia Gallegos Guajardo ORCID: 0000-0001-5678-2353

Universidad de Monterrey, México

Mercedes Gabriela Orozco Solis** ORCID: 0000-0002-0648-8233

Héctor Rubén Bravo Andrade ORCID: 0000-0002-0498-8410 Universidad de Guadalajara, México

Recibido: 17 de enero de 2018 Revisado: 6 de marzo de 2018 Aceptado: 4 de abril de 2018

\section{Resumen}

El objetivo de este estudio fue identificar el nivel predictivo de las competencias socioemocionales sobre la resiliencia, por lo que se aplicaron el Inventario de Competencias Socioemocionales (EQi-YV) y la Escala de Resiliencia para Adolescentes (READ) a una muestra de 840 adolescentes mexicanos. Los resultados obtenidos a través de un análisis de ecuaciones estructurales, señalan que las habilidades de adaptabilidad, así como el optimismo y el autoconcepto, favorecen la competencia personal. Mientras que las competencias interpersonales y la capacidad para generar emociones positivas, predicen las competencias sociales asociadas a la resiliencia. Destaca que el manejo de emociones negativas como el enojo, pierde significancia en el modelo. Los resultados se discuten a la luz de la importancia del desarrollo positivo del adolescente.

Palabras clave: inteligencia emocional, competencias socioemocionales, resiliencia, desarrollo positivo, adolescentes.

Artículo de investigación. Citar como: Ruvalcaba, N., Gallegos, J., Orozco, M., \& Bravo, H. (2019). Validez predictiva de las competencias socioemocionales sobre la resiliencia en adolescentes mexicanos. Diversitas: Perspectivas en Psicología, 15(1), 87-99. DOI: https://doi. org/10.15332/s1794-9998.2019.0001.07

Correspondencia: Dirección postal: Sierra Mojada 950, Independencia Oriente, 44340 Guadalajara, Jalisco. Centro Universitario de Ciencias de la Salud, Universidad de Guadalajara, México. Correo electrónico: mercedes.orozco@cucs.udg.mx 


\section{Abstract}

The purpose of this study was to identify the predictive level of socio-emotional skills on resilience. The Inventory of Socioemotional Skills (EQi-YV) and the Resilience Scale for Adolescents (READ) were applied to a sample of 840 Mexican adolescents. The results obtained through a structural equation analysis, indicated that adaptability, optimism and selfsteem, promote personal competences, while interpersonal skills and generating a positive mood, facilitate social competence associated with resilience. The management of negative emotions, like anger, lost significance in the model. The results are discussed in light of the importance of positive adolescent development.

Keywords: emotional intelligence, social-emotional skills, resilience, positive development, adolescents.

El panorama de salud mental en los adolescentes es inquietante, considerando que un alto porcentaje cubre los criterios para el diagnóstico de problemas de salud mental como ansiedad, depresión, impulsividad y abuso de substancias (Benjet, Borges, Medina-Mora, Zambrano, \& Aguilar-Gaxiola, 2009; Moral \& Ortiz, 2011; Ruvalcaba, Gallegos, Flores, \& Fulquez, 2013; Villatoro et al., 2011). Tomando en cuenta estos datos, es evidente que los adolescentes se encuentran en un importante riesgo de desarrollar problemáticas de distinta índole, las cuales pueden afectar su desarrollo emocional, social y académico (Andrade, Betancourt, Vallejo, Segura, \& Rojas, 2012). Por lo tanto, es necesario que en esta etapa, el individuo cuente con las estrategias de afrontamiento y las habilidades socioemocionales necesarias para responder de manera resiliente frente a las complicaciones que se puedan presentar en su entorno (Cardozo \& Alderete, 2009).

La resiliencia es un recurso que ayuda al ser humano a afrontar las adversidades que se le presentan en la vida cotidiana (Eilersten, Hjemdal, Le, Diseth, \& Reinfjell, 2016; Friborg, Barlaug, Martinussen, Rosenvinge, \& Hjemdal, 2005). La definición de resiliencia es un tema que no ha alcanzado consenso dentro de la literatura, presentando un creciente número de propuestas conforme aumentan las investigaciones al respecto (Pinto, 2014; Windle, 2011). En la definición general, suele describirse como la capacidad de supe- rar los eventos adversos y ser capaz de tener un desarrollo exitoso a pesar de las circunstancias (Becoña, 2006). Sin embargo, es importante además considerar su acepción como un proceso que necesita de la interacción de diferentes mecanismos, entre ellos la capacidad del sujeto para hacer uso de sus factores protectores, conocida como autoeficacia (Friborg, Hjemdal, Rosenvinge, \& Martinussen, 2003; García del Castillo, García del Castillo-López, López-Sánchez, \& Dias, 2015). En este sentido, la resiliencia puede ser considerada como un proceso dinámico, donde influyen aspectos ambientales y personales (internos y externos), que interactúan para ayudar a los adolescentes a adaptarse de manera positiva en situaciones adversas (Hjemdal, Friborg, Stiles, Rosenvinge \& Martinussen, 2006; Cardozo \& Alderete, 2009). Tres son los factores que deben estar presentes en toda definición de resiliencia: la existencia de una adversidad, la presencia de recursos para contrarrestar los efectos de la adversidad y una adaptación positiva (Windle, 2011).

El papel que juega la resiliencia en la vida del ser humano, y particularmente del adolescente, es un tema de relevancia actual, considerando que representa un elemento que puede prevenir la generación de distintas problemáticas. Académicamente, se ha reportado que contar con una respuesta resiliente, favorece un mejor rendimiento académico y disminuye la posibilidad de abandono escolar, protegiendo al estudiante de los 
contextos escolares y amistades de riesgo (Gaxiola, González, \& Contreras, 2012; Gaxiola, González, \& Gaxiola, 2013). Asimismo, algunos autores reportan que existe una correlación negativa entre la resiliencia y los síntomas psiquiátricos. Así, se reporta que a mayor resiliencia las problemáticas como depresión, ansiedad y estrés disminuyen, presentándose como un factor protector frente a fenómenos como el suicidio (Hjemdal, Vogel, Solem, Hagen, \& Stiles, 2011; Sánchez-Teruel \& Robles-Bello, 2014).

La capacidad de responder de manera resiliente frente a la adversidad se encuentra determinada por la presencia de ciertos componentes, los cuales se pueden categorizar en tres áreas: disposiciones personales, cohesión familiar y apoyo social fuera de la familia (Hjemdal, Friborg, \& Stiles, 2012; Limonero, Tomás-Sábado, FernándezCastro, Gómez-Romero, \& Ardilla-Herrero, 2012). Con respecto a los atributos disposicionales del individuo, se identifican factores como habilidades intelectuales, autonomía, autoconfianza, sociabilidad, orientación a metas y adecuadas estrategias de afrontamiento. En el tema de la familia, juegan un papel importante la existencia de relaciones caracterizadas por un trato cálido, cohesión, estructura, soporte emocional, apego positivo y vínculos cercanos. Luego, en la categoría de soporte social externo, se identifican factores como las relaciones interpersonales positivas y la vivencia de experiencias positivas en la escuela y comunidad (Bissonnette, 1998). Otros de los factores que predicen el comportamiento resiliente frente a la adversidad son el auto-concepto, la autorregulación de habilidades cognitivas-emocionales y el soporte social (Eilersten et al., 2016). De tal manera, el ser resiliente implica más que afrontar positivamente la adversidad, involucrando una compleja combinación de las habilidades sociales, con una actitud empática y cooperativa, lo que a su vez favorece que el sujeto pueda acceder a mayor apoyo social (Friborg et al., 2005).

Dentro de esta compleja dinámica, un factor que juega un papel importante en el proceso de respuesta frente a las circunstancias negativas son las habilidades socioemocionales. El adolescente emocional y socialmente inteligente podrá afrontar las situaciones de estrés con mayor éxito, siendo capaz de percibir y valorar sus estados de ánimo, expresar y regular sus emociones, $y$, responder de una manera más asertiva frente a la dificultad (Omar, Paris, Uribe, Almeida, \& Aguiar, 2011).

Las competencias socioemocionales son definidas como un conjunto de capacidades emocionales, individuales e interpersonales, que determinan la habilidad del individuo para responder ante las presiones del medio que lo rodea. Ser emocional y socialmente inteligente significa gestionar con eficacia el cambio personal, social y ambiental de forma realista y flexible, es decir, de afrontar las situaciones inmediatas, resolver problemas y tomar decisiones, mostrando optimismo, actitud positiva y automotivación (Bar-On, 2006). La auto-regulación es uno de los componentes socioemocionales identificados como predictores importantes de resiliencia para ambos sexos, presentándose como un factor de mayor importancia que elementos como la autoestima (Buckner, Mezzacappa, \& Beardslee, 2003). El contar con competencias socioemocionales adecuadas impacta positivamente en la regulación de emociones, el bienestar subjetivo, la resiliencia y la salud (Saarni, 2000; Vidal, 2000). Además, el componente socioemocional influye en otros factores como la autoeficacia, el proceso de aprendizaje, las relaciones interpersonales, la capacidad de resolver problemas, entre otros (Almeida, Omar, Aguiar, \& Carvalho, 2009; Lerner \& Lerner, 2013).

La relación entre las competencias socioemocionales y la resiliencia está identificada claramente por la literatura, a pesar de existir pocos trabajos recientes al respecto (Almeida et al., 2009; Armstrong, Galligan, \& Crirchley, 2011; Phillippe, Lecorrs, \& Beaulieu-Pelletier, 2009; Tugade \& Fredrickson, 2007). Sin embargo, además de la necesidad de actualizar la información científica existente al respecto, es necesario también analizar el papel que los componentes personales pudieran tener como mediadores de la relación entre estas variables. Considerando que pueden funcionar como movilizadores de otro tipo de recursos para hacer frente a la adversidad, y explicar las diferencias de respuesta que se observan de un individuo a otro, aun cuando 
se encuentran en las mismas circunstancias y reciben los mismos apoyos.

En este trabajo en particular, nos centraremos en el concepto de la competencia personal que en el constructo hace específicamente referencia a la autoeficacia, definida como la percepción individual de ser capaz de ejecutar las acciones necesarias para alcanzar el resultado planeado (Bandura, 1993). Es una característica innata que juega un papel importante en el proceso de respuesta frente a las complicaciones de la vida, dado que le permite al individuo tener la percepción de control sobre las amenazas, disminuyendo las respuestas de ansiedad (Dupere, Leventhal \& Vitaro, 2012). Cuando la persona con alta autoeficacia enfrenta una circunstancia negativa, será más capaz de controlar sus pensamientos y perseverar a través de los momentos difíciles, en comparación con otros individuos con menores niveles (Narayanan \& Cheang, 2016). Además, la autoeficacia se relaciona con mayores habilidades de resolución de problemas y una mayor percepción de recursos disponibles (Bilgin, 2011).

Considerando lo mencionado anteriormente, la relación entre las competencias socioemocionales y la resiliencia es un tema de importancia cuando se habla de adolescencia. Es necesario identificar las variables que favorecen una respuesta positiva frente a la adversidad, dado que previenen una diversidad de problemáticas que afectan a los adolescentes en la actualidad. De tal manera, los objetivos de este trabajo fueron analizar las diferencias observadas en las variables de estudio con respecto al género. Por otro lado, identificar las asociaciones entre las competencias socioemocionales (competencias intrapersonales, competencias interpersonales, manejo del enojo, adaptabilidad, emociones positivas, optimismo y autoconcepto) y los componentes de la resiliencia (competencia personal, competencia social, cohesión familiar, recursos sociales y orientación a metas); para posteriormente, explorar el valor predictivo de dichos componentes mediante modelo de ecuaciones estructurales.

En la figura 1 se presenta el modelo hipotético diseñado para este trabajo, en donde se plantea la existencia de una relación predictiva entre las competencias socioemocionales y los componentes de la resiliencia, proponiendo que esta interacción se ve mediada por la habilidad del sujeto para movilizar recursos protectores en sus reacciones frente a la adversidad. 
Figura 1. Modelo hipotético de relación entre competencias socioemocionales y componentes de resiliencia.

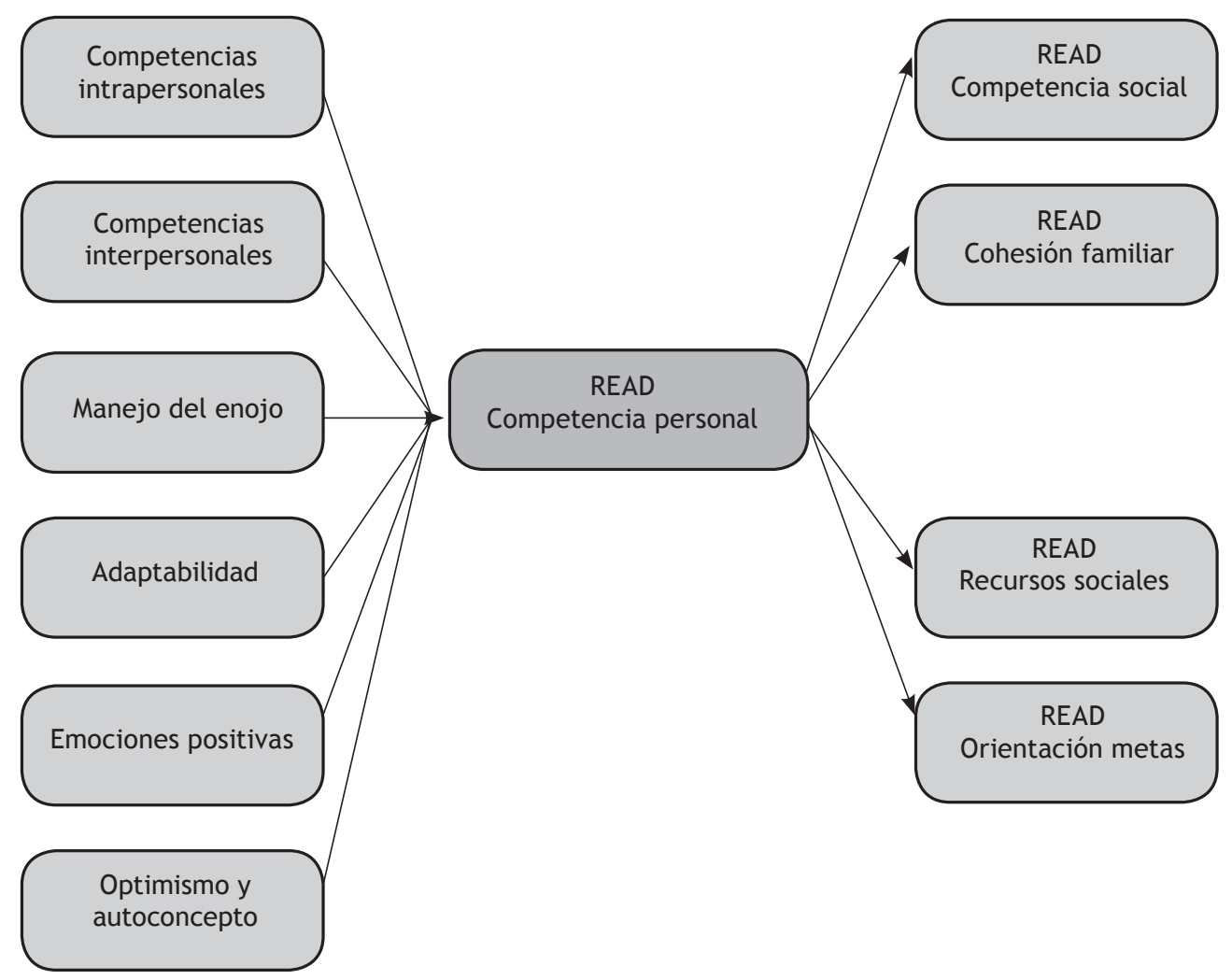

Fuente: Elaboración propia.

\section{Método}

\section{Diseño}

Se trató de un estudio observacional, explicativo y transversal.

\section{Participantes}

La muestra fue elegida por conveniencia y estuvo constituida por 840 adolescentes residentes en la Zona Metropolitana de Guadalajara (México), todos estudiantes de secundaria, con una edad comprendida entre los 12 y los 17 años, con una media de 14.3 años $(D E=1,346)$. Con respecto al género, el $44 \%$ fueron hombres y el $56 \%$ mujeres.

\section{Instrumentos}

\section{Ficha de datos sociodemográficos}

Se realizó un formato de una hoja que recogía información sobre las variables género, edad y grado escolar.

\section{Inventario de Competencias}

\section{Socioemocionales}

Versión para jóvenes (EQ-i, Young Version; Bar-On \& Parker, 2000). La versión adaptada a población mexicana por Ruvalcaba, Gallegos, Lorenzo y Borges (2014), es un instrumento de autoinforme de 48 reactivos en escala tipo Likert que valora seis dimensiones: competencias intrapersonales $(\alpha=0,840)$, competencias interpersonales $(\alpha=0,800)$, manejo del enojo $(\alpha=0,620)$, adaptabilidad $(\alpha=0,830)$, emociones positivas $(\alpha=$ $0,670)$ y optimismo y autoconcepto $(\alpha=0,870)$. 
Escala de Resiliencia para Adolescentes (READ; Hjemdal, Friborg, Stiles, Martinussen et al., 2006)

La validación de esta herramienta con población mexicana fue realizada por Ruvalcaba, Gallegos y Villegas (2015). Es un instrumento de autoinforme que comprende 26 reactivos en escala tipo Likert cuya respuesta oscila del 1 (totalmente desacuerdo) a 5 (totalmente de acuerdo). El instrumento valora cinco dimensiones: competencia personal, que implica autoeficacia $(\alpha=0,649)$, competencia social, que considera la destreza social y flexibilidad en ámbitos sociales $(\alpha=0,730)$, cohesión familiar, que relaciona los valores compartidos en familia para mantener una visión positiva $(\alpha=0,827)$, recursos sociales, es decir el acceso y disponibilidad de soporte externo a la familia $(a=0,700)$, y la orientación a metas, que es el grado en que los individuos identifican posibilidades de alcanzar sus objetivos ( $\alpha=0,600$ ).

\section{Procedimiento}

De manera inicial, se solicitó la firma del consentimiento informado de parte de los directores de los planteles educativos participantes y de los padres de los alumnos seleccionados para participar en la investigación. La aplicación de los instrumentos se llevó a cabo de manera grupal, durante el horario regular de clases, siendo realizada por estudiantes previamente capacitados, de la carrera de Psicología de la Universidad de Guadalajara. En cada sesión se explicaron los objetivos, procedimientos y consideraciones éticas del presente estudio, solicitando un asentimiento de parte de los adolescentes que aceptaran participar en la aplicación. Ninguna remuneración material o académica fue provista para los estudiantes. El análisis de los datos se llevó a cabo con el paquete estadístico SPSS v. 21 y AMOS 16.

\section{Resultados}

En la Tabla 1 se presentan los datos descriptivos obtenidos de las escalas aplicadas. Cabe destacar que los varones presentaron mayores puntajes en cuanto a adaptabilidad y competencia personal, mientras que las mujeres puntuaron más alto en las competencias intra e interpersonales, así como en los recursos sociales disponibles.

Tabla 1. Estadísticos descriptivos para competencias socioemocionales y resiliencia.

\begin{tabular}{lcccccccc}
\hline & \multicolumn{2}{c}{ Hombres } & & \multicolumn{2}{c}{ Mujeres } & \multirow{2}{*}{$F$} & \\
\cline { 2 - 3 } Competencias Socioemocionales & Media & DT & & Media & DT & & \\
\hline Competencias intrapersonales & 2,535 & 0,764 & & 2,628 & 0,811 & 2,873 & 0,090 \\
Competencias interpersonales & 2,983 & 0,531 & & 3,182 & 0,487 & 31,969 & 0,000 \\
Manejo del enojo & 2,295 & 0,564 & & 2,311 & 0,518 & 0,172 & 0,678 \\
Adaptabilidad & 3,021 & 0,531 & & 2,948 & 0,485 & 4,368 & 0,037 \\
Optimismo y Auto concepto & 3,335 & 0,545 & & 3,229 & 0,585 & & 7,251 & 0,007 \\
Emociones Positivas & 2,771 & 0,400 & & 2,866 & 0,397 & 11,736 & 0,001 \\
\hline Resiliencia & & & & & & & \\
\hline Cohesión familiar & 3,962 & 0,716 & & 3,879 & 0,792 & 2,499 & 0,114 \\
Competencia social & 3,887 & 0,645 & & 3,945 & 0,69 & & 1,595 & 0,207 \\
Competencia personal & 3,988 & 0,618 & & 3,894 & 0,659 & 4,541 & 0,033 \\
Recursos sociales & 4,270 & 0,599 & & 4,373 & 0,636 & 5,796 & 0,016 \\
Orientación a metas & 4,152 & 0,612 & & 4,191 & 0,630 & 0,832 & 0,362 \\
\hline
\end{tabular}

Fuente: elaboración propia. 
Posteriormente, se realizaron análisis para identificar las asociaciones entre las variables de este estu- dio, se realizó un análisis de correlación de Pearson, cuyos resultados pueden observarse en la Tabla 2.

Tabla 2. Correlación de Pearson entre las subescalas de las competencias socioemocionales y los componentes de la resiliencia.

\begin{tabular}{lccccc}
\hline & $\begin{array}{c}\text { Cohesión } \\
\text { Familiar }\end{array}$ & $\begin{array}{c}\text { Competencia } \\
\text { Social }\end{array}$ & $\begin{array}{c}\text { Competencia } \\
\text { Personal }\end{array}$ & $\begin{array}{c}\text { Recursos } \\
\text { Sociales }\end{array}$ & $\begin{array}{c}\text { Orientación } \\
\text { a Metas }\end{array}$ \\
\hline Competencias Intrapersonales & $0,254^{* *}$ & $0,399^{* *}$ & $0,290^{* *}$ & $0,271^{* *}$ & $0,158^{* *}$ \\
Competencias Interpersonales & $0,248^{* *}$ & $0,470^{* *}$ & $0,342^{* *}$ & $0,384^{* *}$ & $0,309^{* *}$ \\
Manejo del Enojo & $0,104^{* * *}$ & 0,022 & $0,069^{*}$ & $0,090^{* *}$ & 0,041 \\
Adaptabilidad & $0,330^{* *}$ & $0,389^{* *}$ & $0,465^{* *}$ & $0,279^{* *}$ & $0,425^{* *}$ \\
Emociones Positivas & $0,386^{* *}$ & $0,464^{* *}$ & $0,380^{* *}$ & $0,453^{* *}$ & $0,300^{* *}$ \\
Optimismo y Autoconcepto & $0,400^{* * *}$ & $0,358^{* *}$ & $0,528^{* *}$ & $0,389^{* *}$ & $0,387^{* *}$ \\
\hline
\end{tabular}

${ }^{* *} p<0,01,{ }^{*} p<0,05$

Fuente: elaboración propia.

Con la intención de probar el modelo hipotético, se procedió al análisis de ecuaciones estructurales. Los resultados indicaron que el modelo inicial podría ser mejorado. Considerando la posibilidad de que las competencias socioemocionales pudieran estar también influyendo en otras subescalas de la prueba de resiliencia, se procedió a incluir las competencias sociales al modelo y se eliminaron aquellas relaciones que perdieron significancia (modelo 2). Los índices de ajuste no mejoraron y se observó que la validez predictiva de la variable "manejo del estrés" perdió significancia tanto para la "competencia personal" como para la "competencia social", por lo que se decidió retirarla (mo- delo 3). Finalmente se procedió a explorar la validez predictiva de la "competencia personal" sobre la "competencia social", al hacer esto, los índices de ajuste mejoraron considerablemente (tabla 3).

Como se observa en la Figura 2, fueron la "adaptabilidad" y el "estado de ánimo" ("optimismo" y "autoconcepto"), las competencias socioemocionales que presentaron una validez predictiva de la "competencia personal de resiliencia". Por su parte, las competencias interpersonales y la capacidad para generar emociones positivas, fueron las que arrojaron un mayor peso predictivo sobre la "competencia social", componente de la resiliencia.

Tabla 3. Índices de ajuste del modelo de ecuaciones estructurales.

\begin{tabular}{cccccccccccc}
\hline & & & & \multicolumn{4}{c}{ Índices de ajuste absoluto } & \multicolumn{3}{c}{ Índices de ajuste incremental } \\
\cline { 7 - 13 } Modelo & X2 & gl & x2/gl & GFI & AGF I & RMR & RMSE A & NFI & TLI & CFI & IFI \\
\hline 1 & 673,117 & 30 & 22,43 & 0,856 & 0,684 & 10,258 & 0,160 & 0,795 & 0,635 & 0,801 & 0,803 \\
2 & 43,434 & 10 & 4,34 & 0,988 & 0,956 & 0,476 & 0,063 & 0,982 & 0,961 & 0,986 & 0,986 \\
3 & 24,245 & 5 & 4,84 & 0,992 & 0,954 & 0,294 & 0,068 & 0,989 & 0,962 & 0,991 & 0,991 \\
4 & 7,639 & 3 & 2,54 & 0,997 & 0,979 & 0,196 & 0,043 & 0,996 & 0,980 & 0,997 & 0,980 \\
\hline
\end{tabular}

Fuente: elaboración propia. 
Figura 2. Modelo final.

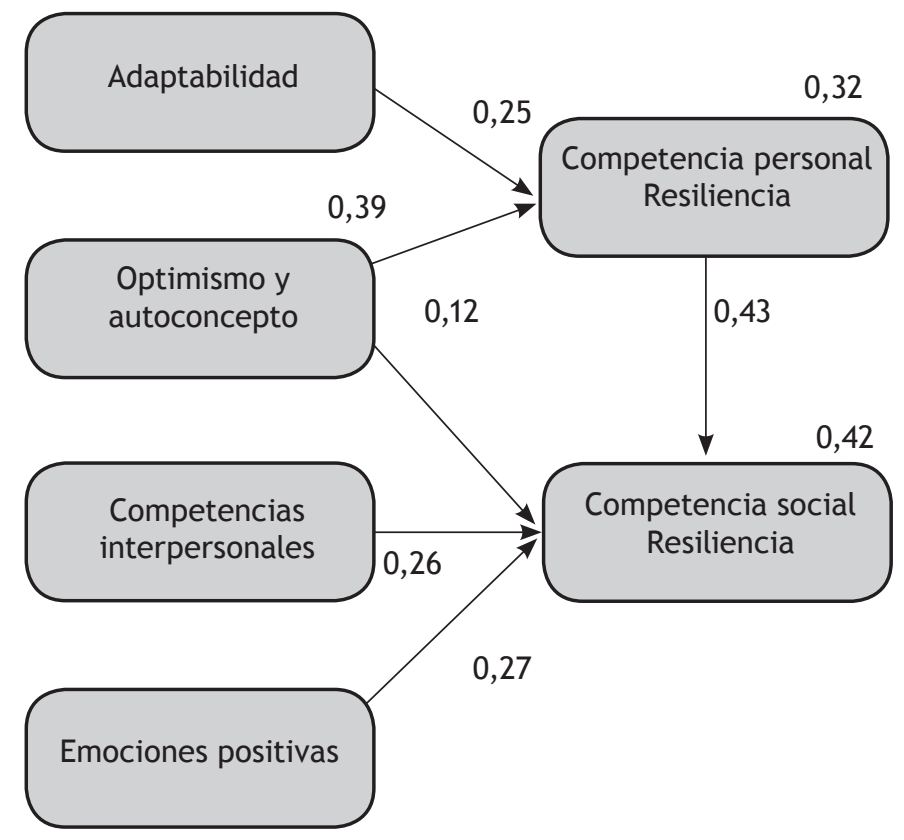

Fuente: elaboración propia.

\section{Discusión y Conclusiones}

Los objetivos de este trabajo fueron analizar las diferencias observadas en las variables de estudio, comparando para el género. Así mismo, identificar las asociaciones entre las competencias socioemocionales (competencias intrapersonales, competencias interpersonales, manejo del enojo, adaptabilidad, emociones positivas, optimismo y autoconcepto) y los componentes de la resiliencia (competencia personal, competencia social, cohesión familiar, recursos sociales y orientación a metas); para posteriormente, explorar el valor predictivo de dichos componentes mediante modelo de ecuaciones estructurales, mediando la relación por la competencia personal (autoeficacia).

Con respecto a las diferencias por género, se encontró que, los varones puntuaron más alto en adaptabilidad y competencia personal (autoeficacia), mientras que las mujeres obtuvieron mejores puntajes en las competencias intra e interpersonales, así como para los recursos sociales disponibles. Es probable que estos resultados estén siendo influidos por una cuestión de perspectiva de género, al hablar de inteligencia emocional, fortalezas y otros temas relacionados con el abordaje de los cambios de la vida, suelen encontrarse diferencias relativamente generalizadas entre ambos sexos (Ovejero \& Cardenal, 2015). Esto coincide con lo reportado en otros trabajos donde se encuentra que los adolescentes varones muestran mayor resiliencia en cuestiones de independencia, mientras que las adolescentes lo hacen en cuanto al apoyo social (González, Valdez, \& Zavala, 2008). Asimismo, en un trabajo realizado con estudiantes universitarios españoles, se encontraron las mismas diferencias al hablar de inteligencia emocional, identificando los hombres como más adaptables para afrontar las situaciones de estrés y a las mujeres como poseedoras de mayores recursos internos y sociales para el abordaje de este tipo de circunstancias (Clariana, Cladellas, Badia, \& Gotzens, 2011; Extremera, Durán, \& Rey, 2007).

Se encontraron asociaciones positivas y estadísticamente significativas entre la mayoría de las subescalas del EQ-i YV y el READ, lo cual coincide con lo encontrado por diversos autores y señala a la inteligencia emocional como precursora de la 
resiliencia (Almeida et al., 2009; Kinman \& Grant, 2011; Lerner \& Lerner, 2013; Limonero et al., 2012; Ruvalcaba et al., 2015; Saarni, 2000).

Al identificar la validez predictiva de las competencias socioemocionales sobre la resiliencia, se encontró que las habilidades interpersonales favorecen la competencia social de la resiliencia. Esto sugiere que habilidades como la empatía, la responsabilidad social y el manejo efectivo de relaciones interpersonales, son factores que en parte predicen la destreza con la que el individuo se maneja socialmente, la flexibilidad que muestra en sus relaciones interpersonales y con la que afronta el cambio. Resultados similares han sido encontrados en diversas investigaciones (ej., Kinman \& Grant, 2011; Omar et al., 2011), enfatizando la importancia de desarrollar intervenciones que promuevan un desarrollo emocional y social positivo en el adolescente desde las etapas más tempranas posibles.

De manera similar, los resultados del modelo de ecuaciones estructurales muestran que la adaptabilidad y la generación de un estado anímico positivo facilitan la competencia personal asociada con la resiliencia. Además, las emociones positivas mantuvieron también un papel importante en la predicción de la competencia social de resiliencia. Este resultado nos habla de la relevancia que parece tener la generación y experimentación de emociones positivas en los adolescentes, manteniendo un papel predictivo que supera el manejo de las emociones negativas, como enojo o estrés. Esto sugiere que, no es particularmente el manejo de situaciones adversas y emociones negativas lo que fomenta la resiliencia, sino la capacidad de experimentar emociones positivas disfrutando de las experiencias de manera cotidiana y, aun cuando se han experimentado eventos negativos.

Considerando lo anterior, la vivencia de emociones positivas, tiene un efecto importante en la respuesta resiliente que se genera al experimentar circunstancias adversas, tomando en cuenta que fortalece los recursos de afrontamiento resiliente y disminuye el impacto de las emociones negativas derivadas del estrés cotidiano en los adultos que cuentan con alto nivel de resiliencia (Armstrong et al., 2011; Fredickson, 2005; Phillippe et al.,
2009; Tugade \& Fredrickson 2007). Estos hallazgos están en concordancia con los trabajos que señalan el efecto undoing de las emociones positivas (Fredrickson, 2005; Fredickson, Mancurso, Branigan, \& Tugade 2000) y con estudios específicos como los de Armstrong et al. (2011), Greco, Morelato e Ison (2006), Fredrickson y Joiner (2002), Tugade y Fredrickson (2007) y Almeida et al. (2009). Cabe destacar que, además de lo señalado, las emociones positivas presentan un efecto predictor contundente en el bienestar subjetivo, optimismo, satisfacción con la vida y autoestima de una persona (Phillippe et al., 2009; Tugade \& Fredrickson, 2007), por lo que es también importante incluir estrategias para aumentar emociones positivas dentro de los programas desarrollados para el adolescente.

Desde el punto de vista teórico, el encontrar que las emociones positivas mantienen una relación tan importante con la competencia social de la resiliencia, nos direcciona a los trabajos iniciales de desarrollo de la Psicología Positiva. En donde, autores como Seligman \& Csikszentmihalyi (2000), retomaban la idea de que, no es suficiente estudiar los efectos adversos que tiene una experiencia negativa en el individuo para ayudarle a superarla o prevenirla, sino que, es necesario resaltar el papel que factores como las emociones positivas, juegan como generadoras de fortalezas y bienestar. Es imposible evitar que el ser humano se enfrente a cambios constantes y situaciones adversas, en etapas tan críticas como lo es la adolescencia. Sin embargo, la clave está en entender que es necesario controlar las emociones negativas que se generan a partir de ellas, pero es más importante todavía, desarrollar en el adolescente la habilidad para cultivar emociones positivas que promuevan una mejor respuesta frente a la circunstancia experimentada.

Además de lo anterior, los resultados de este trabajo subrayan la necesidad de hacer énfasis en la promoción temprana de otros factores protectores que favorezcan el desarrollo positivo del adolescente, como los son: las competencias, la confianza en sí mismo, la creación de vínculos interpersonales sólidos, la integridad, y la compasión (Lerner \& Lerner, 2013). Estos factores protectores facilitan la resiliencia y aminoran el impacto sobre la salud mental que pueden afectar los factores de riesgo a 
los que se ven sometidos una gran parte de los adolescentes en México, como por ejemplo la pobreza, violencia, deserción escolar, y la accesibilidad a sustancias adictivas. La integración de la promoción de estos factores protectores se vuelve cada vez más urgente dado el panorama que atraviesa el país, en donde el $7,2 \%$ de la población adolescente en México ha padecido de depresión (Benjet et al., 2009) y aproximadamente el $30 \%$ ha reportado síntomas de ansiedad, los cuales acompañan a otras problemáticas como las adicciones y la violencia (González-Forteza, Arana, \& Jiménez, 2008).

Finalmente, y como prospectiva de investigación, se sugiere la realización de estudios longitudinales que incluyan instrumentos que evalúen tanto factores de riesgo, como ansiedad y depresión, así como indicadores de salud mental positiva como los niveles de bienestar, optimismo y felicidad. El incluir múltiples informantes como padres de familia y maestros, y complementar la información con entrevistas cualitativas o grupos focales sería también muy útil para comprender mejor el cómo interactúan estas variables.

\section{Referencias}

Almeida, S., Omar, A., Aguiar, M., \& Carvalho, S. R. (2009). Resiliencia e inteligencia emocional en adolescentes: Un estudio de Brasil, Argentina y México. En Conselho Regional de Psicología. Resumos de comunicaciones científicas. VI Congresso Norte Nordeste de Psicología. Belém: UFPará.

Andrade, P., Betancourt, D., Vallejo, A., Segura, B., \& Rojas, R.M. (2012). Prácticas parentales y sintomatología depresiva en adolescentes. Salud Mental, 35, 29-36.

Armstrong, A., Galligan, R., \& Critchley, C. (2011). Emotional intelligence and psychological resilence to negative life events. Personality and Individual Differences, 51(3), 331-336. Doi: https://doi.org/10.1016/j.paid.2011.03.025

Bandura, A. (1993). Perceived self-efficacy in cognitive development and functioning. Educational
Psychologist, 28(2), 117-148. Doi: https://doi. org/10.1207/s15326985ep2802_3

Bar-On, R. (2006) The Bar-On Model of emotional-social intelligence (ESI). Psicothema, 18(Supl), 13-25.

Bar-On, R. \& Parker, J. (2000). The Bar-On Emotional Quotient Inventory: Youth Version (EQ-i: YV) Technical Manual. Toronto, Canada: Multi-Health Systems, Inc.

Becoña, E. (2006). Resiliencia: Definición, características y utilidad del concepto. Revista de Psicopatología y Psicología Clínica, 11(3), 125-146.

Benjet, C., Borges, G., Medina-Mora, M. E., Zambrano, J., \& Aguilar-Gaxiola, S. (2009). Youth mental health in a populous city of the developing world: Results from the Mexican adolescent health survey. Journal of Child Psychology and Psychiatry, 50(4), 386-395. Doi: https://doi. org/10.1111/j.1469-7610.2008.01962.x

Bilgin, M. (2011). Relations among proposed predictors and outcomes of social Self-efficacy in Turkish late adolescents. Eğitim Fakültesi Dergisi, 40(1), 1-18.

Bissonnette, M. (1998). Optimism, Hardiness, and Resiliency: A review of the Literature. [Documento electrónico] Recuperado de: http://www.reachinginreachingout.com/documents/Optimism\%20Hardiness\%20and\%20 Resiliency.pdf

Buckner, J., Mezzacappa, E., \& Beardslee, W. (2003). Characteristics of resilient youths living in poverty: The role of self-regulatory processes. Development and Psychopathology, 15, 139-161. Doi: https://doi.org/10.1017. S0954579403000087

Cardozo, G. \& Alderete, A.M. (2009). Adolescentes en riesgo psicosocial y resiliencia. Psicología desde el Caribe, 23(1), 148-182.

Clariana, M., Cladellas, R., Badia, M., \& Gotzens, C. (2011). La influencia del género en variables de 
la personalidad que condicionan el aprendizaje: inteligencia emocional y procrastinación académica. Revista Electrónica Interuniversitaria de Formación del Profesorado, 14(3), 87-96.

Dupere, V., Leventhal, T., \& Vitaro, F. (2012). Neighborhood processes, self-efficacy and adolescent mental health. Journal of Health and Social Behavior, 53(2), 183-198. Doi: https:// doi.org/10.1177/0022146512442676

Eilersten, M.E., Hjemdal, O., Le, T.T., Diseth, T., \& Reinfjell, T. (2016). Resilience factors play an important role in the mental health of parents when children survive acute lymphoblastic leukemia. Foundation Acta Paediatrica, 105(1), 3034. Doi: https://doi.org/10.1111/apa.13232

Extremera, N., Durán, A., \& Rey, L. (2007). Inteligencia emocional y su relación con los niveles de burnout, engagement y estrés en estudiantes universitarios. Revista de Educación, 342, 239-256.

Fredrickson, B. (2005). Positive emotions. In Snyder, C., \& Lopez, S. (Ed.), Handbook of Positive Psychology (120-135). Oxford: Oxford University Press.

Fredrickson, B. \& Joiner, T. (2002). Positive emotions trigger upward spirals toward emotional wellbeing. Psychological Science, 13(2), 172-175. Doi: https://doi.org/10.1111/1467-9280.00431

Fredickson, B., Mancurso, R., Branigan, C., \& Tugade, M. (2000). The undoing effect of positive emotions. Motivations and Emotions, 24(4), 237-258. Doi: https://doi.org/10.1023/A:1010796329158

Friborg, O., Barlaug, D., Martinussen, M., Rosenvinve, J., \& Hjemdal, O. (2005). Resilience in relation to personality and intelligence. International Journal of Methods in Psychiatric Research, 14(1), 29-42. Doi: https:// doi.org/10.1002/mpr.15

Friborg, O., Hjemdal, O., Rosenvinge, J., \& Martinussen, M. (2003). A new rating scale for adult resilience: What are the central protective resources behind healthy adjustment?
International Journal of Methods in Psychiatric Research, 12(2), 65-76. Doi: https://doi. org/10.1002/mpr.143

García del Castillo, J. A., García del Castillo-López, Á., López-Sánchez, C., \& Dias, P. C. (2015). Conceptualización teórica de la resiliencia psicosocial y su relación con la salud. Health \& Addictions, 16(1), 59-68.

Gaxiola, J., González, S., \& Contreras, Z. (2012). Influencia de la resiliencia, metas y contexto social en el rendimiento académico de bachilleres. Revista Electrónica de Investigación Educativa, 14(1), 164-181.

Gaxiola, J., González, S., \& Gaxiola, E. (2013). Autorregulación, resiliencia y metas educativas: Variables protectoras del rendimiento académico de bachilleres. Revista Colombiana de Psicología, 22(2), 241-252.

González, N., Valdez, J., \& Zavala, Y. (2008). Resiliencia en adolescentes mexicanos. Enseñanza e investigación en Psicología, 13(1), 41-52.

González-Forteza, C., Arana, D., \& Jiménez, A. (2008). Problemática suicida en adolescentes y el contexto escolar: vinculación autogestiva con los servicios de salud mental. Salud Mental, 31(1), 23-27.

Greco, C., Morelato, G., \& Ison, M. (2006). Emociones positivas: Una herramienta para promocionar los procesos de resiliencia infantil. Psicodebate, 7, 81-94. Doi: https://doi. org/10.18682/pd.v7i0.429

Hjemdal, O., Friborg, O., \& Stiles. T.C. (2012). Resilience is a good predictor of hopelessness even after controlling for stressful life events, mood and personality. Scandinavian Journal of Psychology, 53(2), 178-180. Doi: https://doi. org/10.1111/j.1467-9450.2011.00928.x

Hjemdal, O., Friborg, O., Stiles, T., Martinussen, M., \& Rosenvinge, J. (2006). A new scale for adolescents resilience: Grasping the central protective resources behind healthy development. 
Measurement and Evaluation in Counseling and Development, 39, 84-96. Doi: https://doi.org/ 10.1080/07481756.2006.11909791

Hjemdal, O., Friborg, O., Stiles, T., Rosenvinge, J., \& Martinussen, M. (2006). Resilience predicting psychiatric symptoms: a prospective study of protective factors and their role in adjustment to stressful life events. Clinical Psychology and Psychotherapy, 13(3) 194-201. Doi: https://doi. org/10.1002/cpp.488

Hjemdal, O., Vogel, P.A., Solem, S., Hagen, K., \& Stiles, T.C. (2011). The relationship between resilience and levels of anxiety, depression, and obsessive-compulsive symptoms in adolescents. Clinical Psychology and Psychotherapy, 18(4), 314-321. Doi: https:// doi.org/10.1002/cpp.719

Kinman, G. \& Grant, L. (2011). Exploring stress resilience in trainee social workers: The role of emotional and social competencies. British Journal of Social Work, 41(2), 261-275. Doi: https://doi.org/10.1093/bjsw/bcq088

Lerner, R. \& Lerner, J. (2013). The Positive Development of Youth: Comprehensive Findings from the 4-h Study of Positive Youth Development. Massachusetts: Tufts University.

Limonero, J., Tomás-Sábado, J., Fernández-Castro, J., Gómez-Romero, M., \& Ardilla-Herrero, A. (2012). Estrategias de afrontamiento resilientes y regulación emocional: Predictores de satisfacción con la vida. Psicología Conductual, 20(1), 183-196.

Moral, J. \& Ortiz, H. (2011). Un modelo predictivo de conducta disocial por análisis de senderos. Anuario de Psicología Jurídica, 21, 27-40. Doi: https://doi.org/10.5093/jr2011v21a3

Narayanan, S. \& Cheang, A. (2016). The influence of perceived social support and self-efficacy on resilience among first year Malaysian students. Kajian Malaysia, 34(2), 1-23. Doi: https://doi. org $/ 10.21315 / \mathrm{km} 2016.34 .2 .1$
Omar, A., Paris, L., Uribe, H., Ameida, S. H., \& Aguiar, M. (2011). Un modelo explicativo de resiliencia en jóvenes y adolescentes. Psicología em Estudo, 16(2), 269-277. Doi: https://doi. org/10.1590/S1413-737222011000200010

Ovejero, M. \& Cardenal, V. (2015). Las fortalezas humanas desde la perspectiva de género: un estudio exploratorio en población española. Revista Mexicana de Investigación en Psicología, 7(2), 72-92.

Phillippe, F.I., Lecours, S., \& BeaulieuPelletier, G. (2009). Resilience and positive emotions: Examining the role of emotional memories. Journal of Personality, 77(1), 139-176. Doi: https://doi. org/10.1111/j.1467-6494.2008.00541.x

Pinto, C. (2014). Resiliencia Psicológica: Una aproximación hacia su conceptualización, enfoques teóricos y relación con el abuso sexual infantil. Summa Psicológica, 11(2), 19-33. Doi: https:// doi.org/10.18774/448x.2014.11.129

Ruvalcaba, N., Gallegos, J., Flores, A., \& Fulquez, S. (2013). Las competencias socioemocionales como factor protector ante la sintomatología de ansiedad y depresión en adolescentes. Psicogente, 16(29), 55-64.

Ruvalcaba, N., Gallegos, J., Lorenzo, M., \& Borges, A. (2014). Propiedades psicométricas del inventario de competencias socioemocionales para adolescentes (EQi-YV) en población mexicana. Evaluar, 14(1), 1-14.

Ruvalcaba, N.A, Gallegos, J., \& Villegas, D. (2015). Validation of the Resilience Scale for Adolescents (READ) in Mexico. Journal of Behavior, Health, and Social Issues, 6(2), 21-34.

Saarni, C. (2000). Emotional Competence. In Bar On \& Parker (Ed.), Handbook of Emotional Intelligence (68-91). San Francisco: Jossey Bass.

Sánchez-Teruel, D. \& Robles-Bello, M. (2014). Factores protectores que promueven la resi- 
liencia ante el suicidio en adolescentes y jóvenes. Papeles del psicólogo, 35(2), 181-192.

Seligman, M. E. P. \& Csikszentmihalyi, M. (2000). Positive Psychology: An introduction. American Psychologist, 55(1), 5-14. Doi: https://doi. org/10.1037//0003-066X.55.1.5

Tugade, M. \& Fredrickson, B. (2007). Regulation of positive emotions: Emotion regulation strategies that promote resilience. Journal of Happiness Studies, 8(3), 311-333. Doi: https:// doi.org/10.1007/s10902-006-9015-4
Vidal, J. (2000). Apuntes de pisconeuroinmunología. Barcelona: Ediciones UB.

Villatoro, J., Medina, M., Fleiz, C., Moreno, M., Robles, O. ... Buenabad, A. (2012). El consumo de drogas en México: Resultados de la Encuesta Nacional de Adicciones 2011. Salud mental, 35(6), 447-457.

Windle, G. (2011). What is resilence? A review and concept analysis. Reviews in Clinical Gerontology, 21(2), 152-169. Doi: https://doi. org/10.1017/S0959259810000420 